O.M. MATOS

G.A. TORCHIA

G.M. BILMES $3.4, \square$

J.O. TOCHO

H.F. RANEA-SANDOVAL ${ }^{6}$

\section{On the origin of the acoustic signals generated in KDP by pulsed UV laser excitation}

\author{
${ }^{1}$ Departamento de Óptica, Universidad Complutense de Madrid, 28400 Madrid, Spain \\ ${ }^{2}$ Departamento de Física de Materiales C-IV, Universidad Autónoma de Madrid, 28049 Canto Blanco, Madrid, \\ Spain \\ ${ }^{3}$ Facultad de Ingeniería, Universidad Nacional de La Plata, Argentina \\ ${ }^{4}$ Centro de Investigaciones Ópticas (CONICET-CIC), CC 124, 1900 La Plata, Argentina \\ ${ }^{5}$ Departamento de Física, Facultad de Ciencias Exactas, Universidad Nacional de La Plata, Argentina \\ ${ }^{6}$ Instituto de Física 'Arroyo Seco', Facultad de Ciencias Exactas, Universidad Nacional del Centro de la Provincia \\ de Buenos Aires, Pinto 399, B7000GHG Tandil, Argentina
}

\author{
Received: 25 August 2004 / Final version: 10 December 2004 \\ Published online: 24 February 2005 • \\ C) Springer-Verlag 2005
}

ABSTRACT The origin of the acoustic signals that are generated in the bulk of a KDP crystal during irradiation with short UV laser pulses is determined. The generation of these signals by excitation with moderate or high optical fluences is linked to the evolution of the population of point defects that is generated in the crystal by absorption of two UV photons. These defects are bleached due to their efficient linear absorption of $\mathrm{UV}$ radiation, and their non-radiative relaxation is shown to be the origin of the acoustic signals. The rate constants for the different processes involved in both the linear and the non-linear interactions were determined from the experiments presented here. Characteristic values for the quantum efficiency for the generation of defects, $\eta_{\mathrm{F}}=0.95 \pm 0.05$, and for the quantum efficiency for bleaching of defects, $\eta_{\mathrm{B}}=0.065 \pm 0.005$, were obtained for 266-nm laser radiation. The model developed for the intensity of the acoustic signals reproduces the experimental facts with very good accuracy.

PACS 42.65.Ky; 43.35.Ud; 78.20.Hp

\section{Introduction}

Laser-induced generation of acoustic signals in solids has been used as a tool for the study of several physical phenomena [1, 2]. Acoustic signals produced in a potassium dihydrogen phosphate (KDP) crystal after excitation with $\lambda=266-\mathrm{nm}$ pulses were previously demonstrated, and a method for the orientation of non-linear crystals based on the generation of these photoacoustic signals (PS) was proposed in Ref. [3]. PS can be detected with a piezoelectric transducer (PZT) without the need of an amplifier, thus indicating that the conversion efficiency of optical into acoustic energy is fairly high. Recently [4], we have been working on the characterization of PS in KDP showing that transient defects in the crysFax: +54-221-471-2771, E-mail: gabrielb@ciop.unlp.edu.ar tal are responsible for the acoustic emission. After the KDP crystal has been irradiated with UV light, gray spots are formed in the bulk of the crystal indicating that defects have been formed during the laser irradiation. The lifetime of these defects can be easily determined by using a photoacoustic method developed in Ref. [5], instead of the usual optical way.

In Ref. [3], the main features of the mechanism responsible for the signal generation were suggested. When trying to fit the amplitude of the PS generated for different intensities of UV radiation, however, non-physical values for the linear and for the two-photon absorption coefficients were found. In Ref. [5], it has been shown that if $\mathrm{KDP}$ is irradiated simultaneously with 532- and 266-nm radiation, the PS generation is mainly due to the linear absorption of 532-nm radiation by defects that were generated by the UV radiation. This turns out to be a common experimental situation when KDP is used as a non-linear crystal for second-harmonic generation from 532 to $266 \mathrm{~nm}$. In the last few years, KDP crystals were used as a non-linear medium to generate the UV radiation necessary for fusion experiments with inertial confinement, like the Nova laser or the National Ignition Facility at Lawrence Livermore National Laboratory $[6,7]$. Defect characteristics, such as their formation, evolution, and light-absorption mechanisms, are a major concern for fusion experiments.

For UV irradiation, it is accepted that defects are produced by two-photon absorption as an electron-hole pair is produced in the material. The pair relaxes partially and both the electron and the hole can be stabilized in a variety of positive and negative traps, as is common for color centers in alkaline halides. Besides that, there is no definitive identification of the nature of the traps. The involvement of the $\mathrm{PO}_{4} \mathrm{H}_{2}^{-}$group in the optically active centers is generally accepted [8-17]. A hole localized at one oxygen near a proton vacancy in the basic unit $\left(\mathrm{PO}_{4} \mathrm{H}^{-}\right)$, a self-trapped hole at the basic unit $\left(\mathrm{PO}_{4} \mathrm{H}_{2}\right)$, and a neutral hydrogen $\mathrm{H}^{0}$ localized near the basic group $\left(\mathrm{PO}_{4} \mathrm{H}_{3}^{-}\right)$were proposed as the cause of the coloration of KDP in Refs. [8-17].

The generation and bleaching of transient point defects are both associated with different processes involving the 
interaction between radiation and the KDP structure; in this study we analyze their relative contributions to the PS. We developed a model, called the 'two-step' model, in which all possible channels involved in the point-defect dynamics are considered. We have determined the quantum efficiency for formation and bleaching of point defects by means of a fitting procedure with this model using two parameters very well known in the literature. These two parameters provide useful information about the behavior of the PS at different excitation fluences, as a function of time and history of the crystal.

\section{Theoretical model}

\section{Two-step model}

The proposed mechanism for PS generation in a KDP crystal irradiated with pulsed UV light with photons of energy $\hbar \omega$ was developed with the aid of the energy diagram depicted in Fig. 1.

We call it a 'two-step' mechanism because the PS generation is due to the heat released in two interconnected processes: linear and non-linear UV absorption with generation of transient defects (step A) and the later, linear absorption of the defects at the same wavelength (step B).

Energy involved in linear absorption in KDP is relaxed completely in a non-radiative way, while only a fraction of the energy absorbed in the two-photon process contributes to the PS, because part of its energy is consumed generating the defect. This fact is represented in Fig. 1 displaying the ground energy level of the defect inside the gap, $E_{\mathrm{D}}$, relative to the valence band. Transient defects observed in KDP have a lifetime of several seconds, and their relaxation does not contribute to the acoustic signal in cases in which detection is performed with a technique sensitive only to prompt energy dissipation.

Calling $I(x, t)$ the intensity of the UV pulse inside the KDP sample at depth $x$ (measured from the side that is exposed to the laser) and at time $t$, the amount of heat released, after linear and non-linear absorption, in a time window less

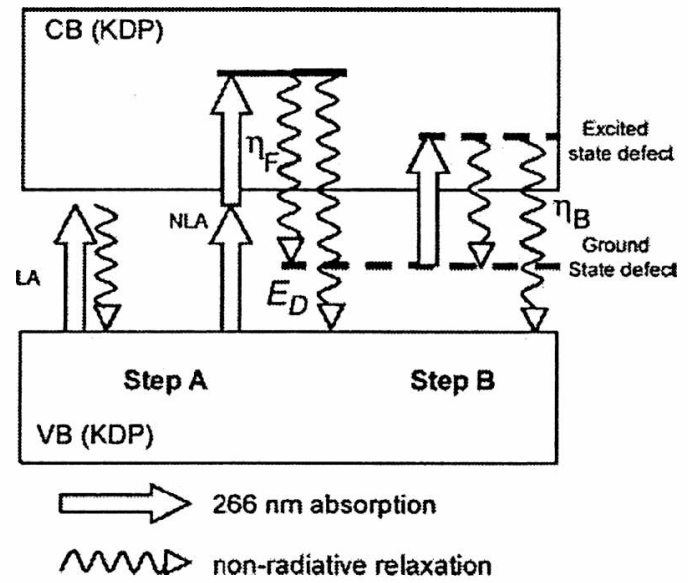

FIGURE 1 Model for photoacoustic signal generation. $L A$ : linear absorption by KDP; $C B$ : conduction band; $V B$ : valence band. Step $A$ : generation of defects by two-photon absorption. Step $B$ : linear absorption by defects. Straight arrou's represent the absorption of one photon. Wavy arrow's represent non-radiative decay than the ground-state lifetime of the defects (step A), can be written as

$H_{\mathrm{A}}=\iint\left[\alpha \hbar \omega+(\beta / 2)\left(2 \hbar \omega-\eta_{\mathrm{F}} E_{\mathrm{D}}\right) I(x, t)\right] I(x, t) \mathrm{d} x \mathrm{~d} t$,

where $\alpha$ and $\beta$ are the linear absorption coefficient and the two-photon absorption coefficient of KDP at frequency $\omega$, respectively, and $\eta_{\mathrm{F}}$ is the quantum efficiency for defect generation by UV two-photon absorption.

In step B a defect absorbs one UV photon that excites it from the ground to an excited state situated within the conduction band. Non-radiative de-excitation from this excited state generates acoustic waves. This de-excitation bleaches the defects with a probability $\eta_{\mathrm{B}}$, causing a PS contribution proportional to $\hbar \omega+E_{\mathrm{D}}$. On the other hand, if the defect is not bleached, the relaxation to its fundamental state proceeds with a probability $1-\eta_{\mathrm{B}}$, resulting in a PS contribution proportional to $\hbar \omega$.

If $N(x, t)$ is the concentration of point defects at a sample depth $x$ at time $t$, the heat produced in step B is

$H_{\mathrm{B}}=\iint \sigma\left(\hbar \omega+\eta_{\mathrm{B}} E_{\mathrm{D}}\right) N(x, t) I(x, t) \mathrm{d} x \mathrm{~d} t$,

where $\sigma$ represents the absorption cross section for defects for light with an energy $\hbar \omega$. The total heat released can be calculated by adding the contributions (Eqs. (1) and (2)), and results in

$$
\begin{gathered}
\mathrm{PS} \propto \iint\left[\alpha \hbar \omega+(\beta / 2)\left(2 \hbar \omega-\eta_{\mathrm{F}} E_{\mathrm{D}}\right) I(x, t)\right. \\
\left.\quad+\sigma\left(\hbar \omega+\eta_{\mathrm{B}} E_{\mathrm{D}}\right) N(x, t)\right] I(x, t) \mathrm{d} x \mathrm{~d} t .
\end{gathered}
$$

It is obvious that Eq. (3) can be integrated to obtain the PS after the space-time dynamics of $N(x, t)$ and of $I(x, t)$ are solved. The equations describing such evolution during each UV pulse are

$$
\begin{aligned}
& \frac{\partial I(x, t)}{\partial x}=-[\alpha+\sigma N(x, t)] I(x, t)-\beta I^{2}(x, t), \\
& \frac{\partial N(x, t)}{\partial t}=\frac{\eta_{\mathrm{F}} \beta I^{2}(x, t)}{2 \hbar \omega}-\frac{\eta_{\mathrm{B}} \sigma N(x, t) I(x, t)}{\hbar \omega} .
\end{aligned}
$$

These equations describe three mechanisms for the attenuation of the incident UV pulse: the linear and the nonlinear absorption by bulk KDP, and the linear absorption by defects.

Defects are generated by two-photon absorption and bleached by radiation with efficiencies $\eta_{\mathrm{F}}$ and $\eta_{\mathrm{B}}$, respectively. Between consecutive UV pulses, defects relax following quite complicated kinetics that is not completely understood. However, experiments with the same sample showed that, after a few seconds, the defect population was reduced only slightly $[4,5]$. Hence, in the analysis of the PS due to the irradiation by a train of UV pulses separated for a few seconds, we can assume that the amount of defects that will survive until the next pulse is the same as that at the end of the previous pulse.

Equations (3) and (4) can be solved numerically to obtain the PS for different UV energies. For all the parameter regions inspected, and for pulse energies of less than $20 \mathrm{~mJ}$ focused on 
a spot of 1-mm diameter, we have found that the UV intensity predicted by our model is attenuated only very slightly along the pump beam. In a first approximation we can, therefore, assume that the intensity is uniform inside the crystal, that is

$I(x, t)=I_{0}, \quad \forall x, t<\tau$,

where $I_{0}$ is the intensity at the surface at $x=0$ and $\tau$ is the duration of the UV pulse. Under this assumption, Eq. (4b) can be solved exactly, giving the density of defects formed during the first pulse, which is

$N_{1}(t)=(P / Q)[1-\exp (-P t)]$

with $P=\eta_{\mathrm{B}} \sigma I_{0} / \hbar \omega$ and $Q=\eta_{\mathrm{F}} \beta \sigma I_{0}^{2} / 2 \hbar \omega$.

The density of defects at the end of the first pulse is $N_{1}=$ $(P / Q)[1-\exp (-P \tau)]$. Thus, the density of defects during the pulse number $n$ in a pulse train is

$N_{n}(t)=N_{1}+N_{n-1} \exp (-P t)$.

The time evolution of the density of defects during the UV irradiation can then be introduced in Eq. (3). Under these conditions the acoustic signal produced by the $n$th UV pulse is described as

$$
\begin{aligned}
\operatorname{PS}(n)= & \operatorname{PS}(n-1)+K(\sigma / Q)\left(\hbar \omega+\eta_{\mathrm{B}} E_{\mathrm{D}}\right) N_{1}^{2} \\
& \times \exp [(2-n) \tau P],
\end{aligned}
$$

where the PS due to the first pulse is

$$
\begin{aligned}
\operatorname{PS}(1)= & K\left\{\alpha \tau \hbar \omega I_{0}+(\beta / 2)\left(2 \hbar \omega-\eta_{\mathrm{F}} E_{\mathrm{D}}\right) \tau I_{0}^{2}+\sigma\left(Q / P^{2}\right)\right. \\
& \left.\times\left(\hbar \omega+\eta_{\mathrm{B}} E_{\mathrm{D}}\right)[\tau P+\exp (-\tau P)-1]\right\} L
\end{aligned}
$$

with $L$ being the length of the crystal and $K$ an arbitrary constant reflecting all the instrumentation parameters such as the efficiency for the transformation from heat to acoustic energy, the sensitivity of the acoustic transducer, etc.

\subsection{Numerical simulations}

To fit experimental results with the model presented above, we face the well-known difficulty shared by all the photoacoustic methods: an absolute calibration of the PS is extremely difficult. Thus, the criterion we used to assess the quality of the fit of our results was the ratio of the predicted photoacoustic signal and its value after an infinite number of pulses, which we call the asymptotic value of the PS, and compare it with the experimentally determined ratio.

We restricted the analysis to the variation of the defectformation probability and the defect-bleaching probability, using accepted values for the linear absorption coefficient $(\alpha$ for KDP and $\sigma$ for the defects) and the two-photon absorption coefficient ( $\beta$ for KDP) at $266 \mathrm{~nm}$, taken from Ref. [16].

The value for $E_{\mathrm{D}}$ can be estimated from the absorption spectrum of the defects. As the defects do not absorb at wavelengths longer than approximately $700 \mathrm{~nm}$, we can safely use $E_{\mathrm{D}}=3 \times 10^{-19} \mathrm{~J}$ over the valence band of $\mathrm{KDP}$, as in Ref. [4].

In Fig. 2 the evolution of the amplitude of the PS is shown as a function of the number of pulses in the train. In each case the PS signal was normalized to its asymptotic

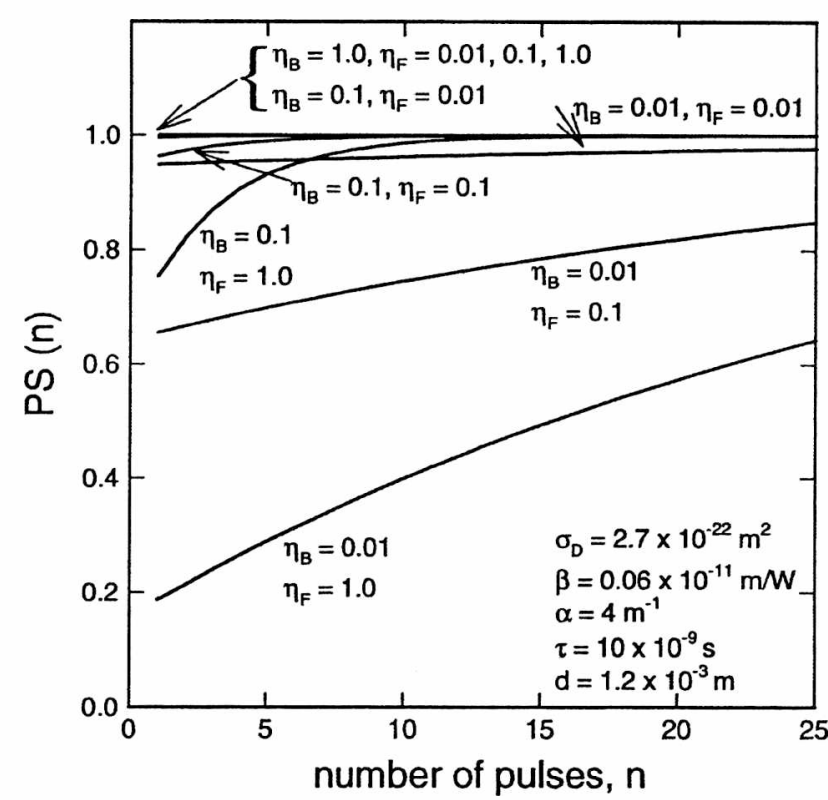

FIGURE 2 Calculated photoacoustic signal amplitude as a function of the number of pulses $(n)$ for different values of $\eta_{\mathrm{F}}$ and $\eta_{\mathrm{B}}$

value that corresponds to the maximum value reached. Errors for the different parameters included in the model were considered.

The results of Eqs. (8) and (9) can be represented for different parameters. It is clear that a no-defects case ( $\eta_{\mathrm{F}}$ close to zero) should give a constant signal from the first pulse on. Similar results should be obtained with strong bleaching $\left(\eta_{\mathrm{B}}\right.$ close to 1) independent of the $\eta_{F}$ value. On the other hand, low bleaching efficiencies correspond to a slow growing of the PS and a much stronger contrast between the first-pulse signal and the asymptotic value will be found.

The calculated amplitude of the PS from the very first pulse and the asymptotic value of the amplitude are shown as a function of the energy of the UV pulses in Fig. 3.

In this case, the PS is normalized to 1 for the asymptotic value for $10-\mathrm{mJ}$ pulse energy. The difference between the PS for the first pulse and the asymptotic value is obvious and depends mainly on the bleaching efficiency.

3

\section{Experimental results}

We have performed experiments to study three aspects of the PS: their dependence on UV laser pulse energy, the relation between the PS and the quantity of defects generated by one pulse, and the accumulative effects of successive pulses on the value of the amplitude of the PS. Excitation was performed using the fourth harmonic of a $\mathrm{Nd}^{+3}$ :YAG laser of 10 ns pulse duration in a train of pulses each separated by $1.5 \mathrm{~s}$. The UV pulse radiation passes through a $1.2-\mathrm{mm}$-diameter pinhole before striking the KDP crystal (see inset in Fig. 4). This was a fresh KDP crystal with a size of $1 \times 1 \times 0.5 \mathrm{~cm}^{3}$, cut for second-harmonic generation of $1060-\mathrm{nm}$ radiation.

The signal from a $200-\mathrm{kHz}$-bandwidth PZT transducer, glued directly to the crystal, was recorded, without amplification, by a digital oscilloscope. The amplitude of the 


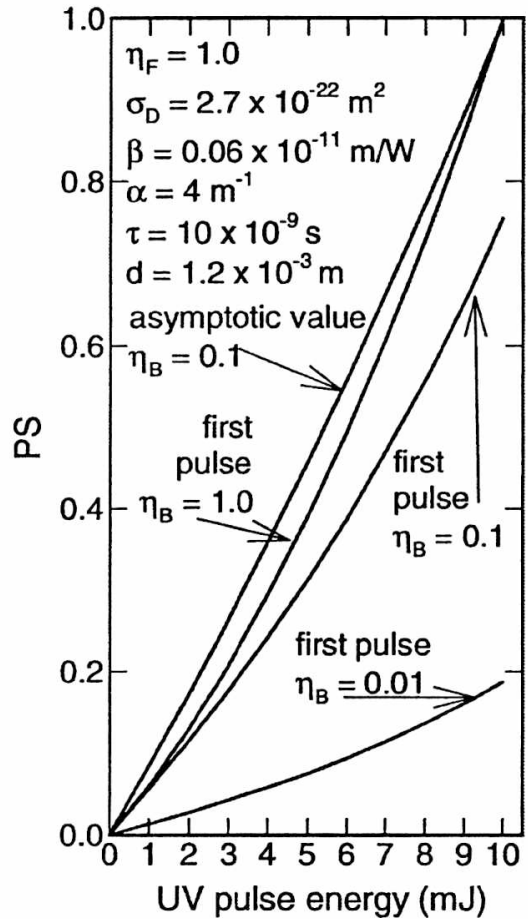

FIGURE 3 Calculated photoacoustic signal amplitude normalized for the asymptotic value for a $10-\mathrm{mJ}$ pulse: comparison between the first-pulse PS and its asymptotic limit, for different values of the bleaching efficiency $\left(\eta_{\mathrm{B}}\right)$

first resonant peak of the ringing was taken as representative of the PS generated in the bulk, as is common.

Figure 4 shows the evolution of the experimental PS (circles) with accumulative UV laser pulses, each one with an energy of $9.9 \mathrm{~mJ}$. The PS grow, approaching an asymptotic level after irradiation with tens of UV pulses.

At the beginning of the experiment the crystal was defectfree, but afterwards the sample had gray defects. This fact, together with the sigmoid character of the curve of Fig. 4,

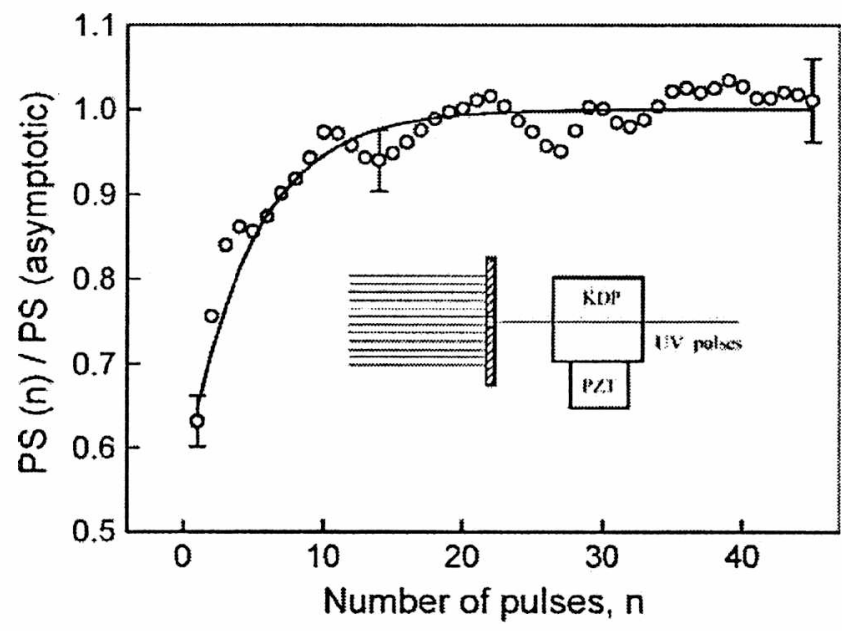

FIGURE 4 Photoacoustic signal as a function of the number of pulses Crystal starts without defects. Signal is normalized for the mean asymptotic value at the maximum energy of $9.9 \mathrm{~mJ}$ per pulse. Full line represents the result of Eq. (6) with the parameters indicated in Table 1. The experimental set-up is indicated in the inset

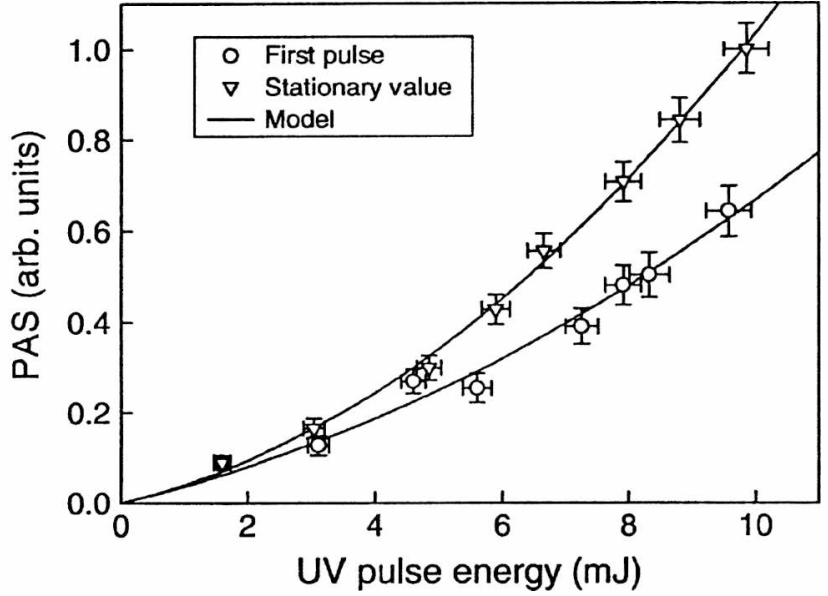

FIGURE 5 Photoacoustic signals corresponding to the first pulse (circle) and the stationary level reached after several pulses 1.5-s apart (square) vs. the energy of the UV pulse. Full lines represent the results of Eqs. (8) and (9), using the parameters of Table 1

suggests that the dependence of the PS on the number of pulses is determined by the growth of the number of defects present in the sample until a stationary level is reached. This asymptotic level is reached when the amount of defects generated by each UV pulse is equal to the number of defects bleached during the pulse.

Figure 5 displays measurements of the amplitude of the normalized PS as a function of the UV pulse energy, performed while maintaining the other experimental conditions unchanged.

Open circles represent the experimental PS observed for single excitation pulses. Open squares represent the stationary level reached after several (typically 40 pulses would be required to reach the asymptotic value) pulses 1.5-s apart. Values for stationary level signals are averaged over several pulses. All the results are normalized to the asymptotic value corresponding to the maximum energy used $(9.9 \mathrm{~mJ})$. There is a growing difference between the PS due to the first pulse and the corresponding stationary value.

The fitting procedure described in Sect. 2.2 yields the continuous lines that are shown in both figures, Figs. 4 and 5 . The complete set of parameters used to draw the fitting curves is summarized in Table 1.

From the fitting procedure we determined the quantum efficiency for the formation of point defects in KDP as $\eta_{\mathrm{F}}=$ $0.95 \pm 0.05$, and for the quantum efficiency for bleaching of defects as $\eta_{B}=0.065 \pm 0.005$ for $266-\mathrm{nm}$ pulsed laser irradiation.

Finally, we used the experimental data depicted in Fig. 5 (circles and triangles) and the fitting procedure based on the above model to explore the influence of the processes involved

\begin{tabular}{ccccc}
\hline $\begin{array}{c}\alpha \\
\left(\mathrm{cm}^{-1}\right)\end{array}$ & $\begin{array}{c}\beta \\
\left(\mathrm{cm}^{-1} / \mathrm{GW}\right)\end{array}$ & $\begin{array}{c}\eta_{\mathrm{F}} \\
(\text { dimensionless })\end{array}$ & $\begin{array}{c}\eta_{\mathrm{B}} \\
(\text { dimensionless })\end{array}$ & $\begin{array}{c}\sigma \\
\left(\times 10^{-18} \mathrm{~cm}^{-2}\right)\end{array}$ \\
\hline 0.03 & 0.085 & 1 & 0.065 & 3.7 \\
\hline
\end{tabular}

TABLE 1 Parameter values used for the best of fits to the experimental results are listed 


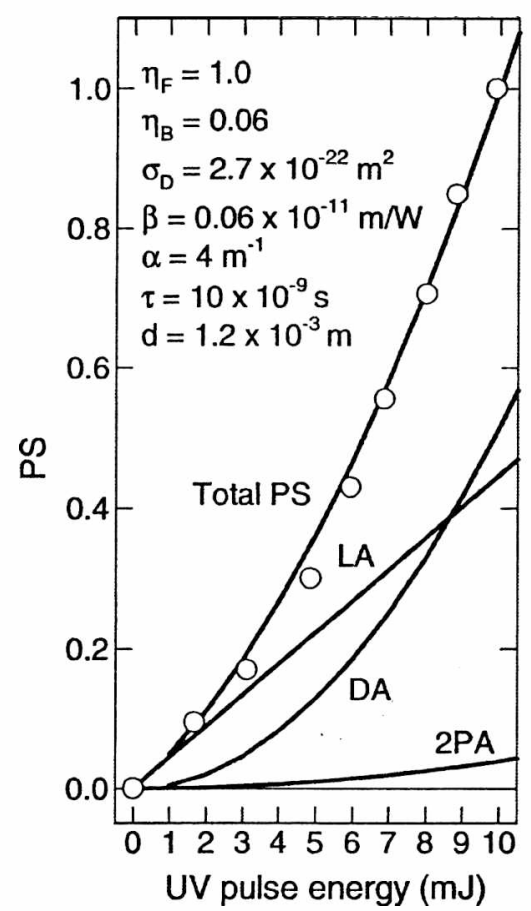

FIGURE 6 Comparison between different model assumptions and experimental data: the open circles are experimental data that correspond to the stationary level reached after several pulses as depicted in Fig. 5. $L A$ : linear absorption of KDP; 2PA: two-photon absorption of KDP; DA: linear absorption of defects

in the generation of the acoustic signal, namely the linear absorption of KDP, the two-photon absorption of KDP, and the linear absorption by defects.

Results are shown in Fig. 6, and the interpretation is as follows: the shown fit indicates that at low laser fluences the signal will be mainly produced by the linear absorption of $\mathrm{KDP}$, while at higher fluences the main contribution to the signal is produced by the defects that relax non-radiatively after a linear absorption process.

\section{4}

\section{Conclusions}

In this study we present, for the first time to our knowledge, an analytic expression for the relative photoacoustic signal generated in KDP irradiated by nanosecond UV pulses. We verify the assumption of a 'two-step' model by fitting the experimental results corresponding to irradiation by one UV pulse on a clear crystal, and the signals corresponding to irradiation by consecutive pulses in a train. The quantum efficiency for the generation of defects by two-photon absorp- tion and the quantum efficiency for the bleaching of defects after absorption of one 266-nm photon are derived with this model from the experimental data. The values we have obtained are in good agreement with those reported in the literature, which were determined with completely independent experiments [11].

We conclude that defect dynamics is the cause of the acoustic signal. Irradiation with relatively low fluence pulses shows that the main acoustic contribution is due to the nonradiative decay of excited states of defects after they absorb the UV radiation. Consequently, the PS intensity as a function of the pump fluence scales with the number of defects.

The predictions from this simple model are almost identical to the ones obtained solving the space-time evolution of the different fields involved, described by Eqs. (1)-(3), and describe with very good accuracy the experimental results. This model can be used as a basis to reproduce the PS produced by phase matching second-harmonic generation from 532 to $266 \mathrm{~nm}$ in KDP crystals. The results presented herein are useful for studies of other crystals used in non-linear processes.

ACKNOWLEDGEMENTS This work was partially supported by FOMEC, CONICET, ANPCYT, UNCPBA, and UNLP. OEM is a Fellow of FOMEC; GMB is a member of the Carrera del Investigador Cientifico CIC-BA; JOT and HFRS are both members of the Carrera del Investigador Científico CONICET

\section{REFERENCES}

1 C.K.N. Patel, A.C. Tam, Rev. Mod. Phys. 53(3), 517 (1981)

2 A.C. Tam, Rev. Mod. Phys. 58(2), 381 (1986)

3 J.O. Tocho, G.M. Bilmes, H.F. Ranea-Sandoval, Appl. Phys. B 69, 473 (1999)

4 O.M. Matos, PhD Thesis, Universidad Nacional de La Plata (2002)

5 O.M. Matos, G.A. Torchia, G.M. Bilmes, J.O. Tocho, Phys. Rev. B 69, 224102 (2004)

6 P. Weiss, Science 275, 1569 (1997)

7 J. Glanz, Science 277, 304 (1997)

8 K. Tsuchida, R. Abe, M. Naito, J. Phys. Soc. Jpn. 35(3), 806 (1973)

9 J.A. McMillan, J.M. Clemens, J. Chem. Phys. 68(9), 3627 (1978)

10 E. Dieguez, J.M. Cabrera, J. Phys. D: Appl. Phys. 14(1), 91 (1981)

11 S.G. Demos, M. Yan, M. Staggs, J.J. De Yoreo, H.B. Radowsky, Appl. Phys. Lett. 72, 2367 (1998)

12 L.N. Ogorodnikov, V.Y. Yakovlev, B.V. Shul'gin, M.K. Satybaldieva, Phys. Solid State 44, 880 (2002)

13 S.D. Setzler, K.T. Stevens, L.E. Halliburton, M. Yan, N.P. Zaintseva. J.J. De Yoreo, Phys. Rev. B 57, 2643 (1998)

14 E. Dieguez, J.M. Cabrera, F. Agulló-López, J. Chem. Phys. 81, 3369 (1984)

15 J.-T. Yu, S.-H. Lou, J. Phys. Soc. Jpn. 62, 3294 (1993)

16 C.D. Marshall, S.A. Payne, M.A. Henesian, J.A. Speath, H.T. Powell, J. Opt. Soc. Am. B 11, 774 (1994)

17 J.E. Davis, R.S. Hughes, H.W.H. Lee, Chem. Phys. Lett. 207, 540 (1993) 